\title{
Spectral Efficiency Improvement of Full-Duplex D2D Communication in Cellular Networks
}

\author{
Salami Bayonle Lukman ${ }^{1}$, Ngyarmunta Alan Audu², Odaba Alphaeus², \\ Ajimah Nnabueze Edmund ${ }^{2}$, Ohemu Monday Fredrick ${ }^{2}$ \\ ${ }^{1}$ Operations Department, Nigerian National Petroleum Corporation (NNPC), Kaduna, Nigeria \\ ${ }^{2}$ Department of Electrical and Electronics Engineering, Air Force Institute of Technology, Kaduna, Nigeria \\ Email address: \\ salamilukman@yahoo.com (S. B. Lukman), an.alan@afit.edu.ng (N. A. Audu), a.odaba@afit.edu.ng (O. Alphaeus), \\ ajimahnnabuezeedmund@gmail.com(A. N. Edmund),monfavour@gmail.com (O.M. Fredrick)
}

\section{To cite this article:}

Salami Bayonle Lukman, Ngyarmunta Alan Audu, Odaba Alphaeus, Ajimah Nnabueze Edmund, Ohemu Monday Fredrick. Spectral Efficiency Improvement of Full-Duplex D2D Communication in Cellular Networks. American Journal of Electromagnetics and Applications. Vol. 8, No. 2, 2020, pp. 46-56. doi: 10.11648/j.ajea.20200802.13

Received: April 1, 2019; Accepted: April 25, 2019; Published: December 22, 2020

\begin{abstract}
Radio spectrum is becoming scarce due to increasing demand for high data rate, mobile communication and everexpanding population with diverse need to always stay interconnected. The cellular network providers and researchers in the academia are continually finding innovative ways to efficiently manage the existing telecommunication infrastructure and plan effective for future expansions and technology. Device to Device (D2D) communication, Full-Duplex (FD) radio, Heterogeneous network are a few of such innovative technologies developed to face the challenges. Device to device communication is one promising technology that is studied for deployment in future network technologies, however it is not without its challenges. Various researches have been carried out and are still being carried out to better understand and improve device to device capabilities. The use of full-duplex radios is an area of study with capability for improving device to device communication due to recent development in full-duplex radio although its major drawback is limited self-interference cancellation abilities to be deployed in large transmit power system. This research presents the practicality of deploying existing FD radios in device to device communication and simulate the amount of self-interference cancellation required using MATLAB for effective use with device to device. Two interference management schemes were implemented to improve the performance of FD-D2D communication, first power control scheme was developed to mitigate interference between D2D and base station in uplink resource sharing, Interference Limited Area (ILA) method was adapted to deal with interference between D2D and cellular user Uplink and Downlink transmission. The performance between 75dB to 110dB Self-Interference (SI) cancellation was carried out. The result was compared to conventional cellular and Half-Duplex D2D communication to estimate the improvement offered on spectral efficiency. The work has improved on achieving almost 100\% spectral efficiency thereby improving the Quality of Service (QoS) for cellular network
\end{abstract}

Keywords: Device-to-Device, Full-duplex, Communication, Spectrum, Self-interference, Cellular, Network, Half-duplex, Spectrum, Wireless

\section{Introduction}

Increasing demand for wireless communications services is leading to the congestion of radio spectrum. Since radio spectrum is an expensive and scarce resource, better utilization of radio resources becomes crucial. Increasing demand for wireless communication requires new solutions and technologies to provide services for end users. With limitations on radio spectrum and the need to support very large number of users and rich multimedia services like high quality video delivery, new technologies are required. Technologies like multiple-input multiple-output (MIMO) systems, cognitive radio, large antenna arrays, in-band fullduplex (FD) radios, device to device communication (D2D) are among new paradigms that are studied for increasing spectral efficiency of wireless systems.

Full-duplex radio design has gained a lot of interest 
recently because of its potential to double the spectrum efficiency of the systems. In conventional two-way wireless communication systems, one node cannot transmit and receive simultaneously on the same frequency band, because the interference generated by outgoing signals can easily overwhelm the incoming signals that are much weaker, this is called self-interference effect. Self-interference can be up to millions of times stronger that the signal of interest, so selfinterference will make it impossible to recover the desired signal [1]. Modeling self-interference and solving this problem will lead to doubling the radio spectrum. The envisaged value of FD radio has led to several full-duplex radio designs, systems like antenna cancelation, balancedunbalanced transformer (BALUN), two stage antenna cancelations, known as full-duplex MIMO (MIDU), more recently single antenna full-duplex radios and full-duplex MIMO [2] are among attempts to build full-duplex radios.

So far $110 \mathrm{~dB}$ of self-interference cancelation is possible for single antenna and multi antenna systems [3-4]. Considering these achievements, investigation of possible application areas of available full-duplex radios becomes an interesting topic. Most of the researches in this area are focused on full-duplex relaying or considering ideal fullduplex systems with perfect self-interference cancelation in cellular systems.

Device to device communication is also seen as a new technology that can improve the system performance and has wide application areas like cellular offloading, machine-tomachine (M2M) communication, video delivery, relaying, location-based services. In D2D, users in close proximity communicate directly without going through the base station (BS) also referred to as enhance NodeB (eNB). D2D users can communicate using unlicensed bands (out-band D2D) bands such as WiFi or ZigBee, which has the problem of uncontrolled interferences or licensed bands (in-band D2D) [5]. In in-band, while using licensed radio resources D2D pair can use its own dedicated resource (overlay) or share the radio resources with one or more cellular users (underlay). While D2D is sharing the resources with other cellular users, both cellular users and D2D pair will face interference from this resource sharing [6]. Figure 1 below illustrates classification of D2D communication.

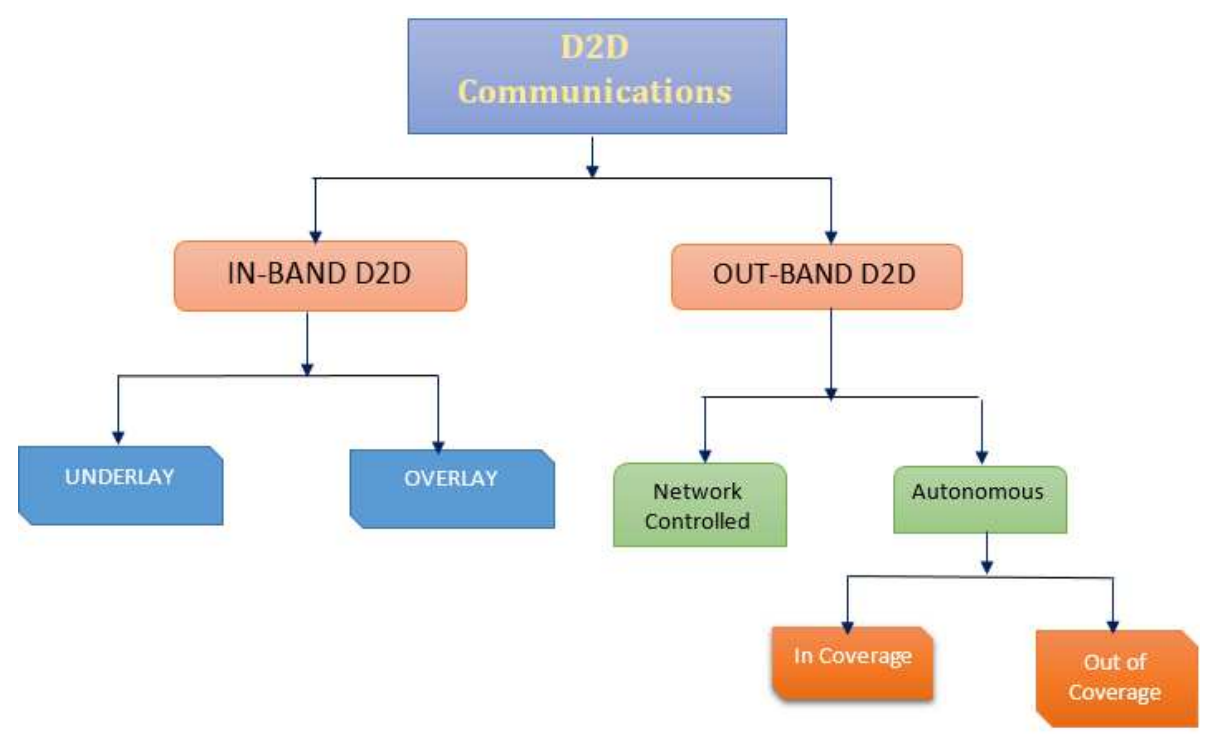

Figure 1. Classification of D2D communication.

The idea of co-sharing the same radio resources between D2D and cellular users leads to higher spectral efficiency. But sharing uplink or downlink resources between cellular and D2D users will result in interference between them [7].

Dealing with this interference is one of the most crucial problems that needs to be addressed in $\mathrm{D} 2 \mathrm{D}$ communications to be able to harness its full benefits. Several interference management methods to deal with this problem are considered in these studies [8-11]. The prospect of combining the advantages offered by full duplex radios with D2D communication to improve spectral efficiency in cellular network is the focus of this work.

With the ever-increasing demand for high data transmission rates is continuously fueled by the relentless growth of wireless data traffic volume due to the rapidly expanding population of broadband users and new emerging applications, such as mobile social networks and electronic commerce. However, available spectrum for wireless communications has already been pushed to the brink of exhaustion. Hence, new and disruptive techniques are crucial to meet this growing mobile traffic demand. As a result, heterogeneous networks (HetNets) [12], full duplex (FD) wireless transmission, and device to device (D2D) communications have been proposed to improve spectrum efficiency and deal with spectrum limitation. FD communications allow a user equipment (UE) to transmit and receive signals at the same time over the same frequency, and thus can potentially double spectral efficiency [13]. However, it has long been regarded as impractical in the past due to strong self-interference (SI). The critical challenge to exploit FD wireless transceivers lies in the huge power discrepancy between its own transmit and receive signal powers, with a 
ratio generally exceeding $100 \mathrm{~dB}$. Therefore, reducing transmit power is a direct way to alleviate SI. On the other hand, full-duplex communication technique has attracted much research interest. The full-duplex communication has the potential to efficiently and significantly improve the network spectral efficiency by using the same frequency band/time slot for both forward and reverse links [14]. Due to the promising potential of device-to-device communication and full-duplex communication techniques, the combination of these two techniques will probably become trend in future cellular networks to solve the critical problem of spectrum scarcity by providing a better utilization. In this work, we propose adopting a Power control scheme to manage interference on the base station for uplink resource sharing in device to device transmitters, and Interference limited area (ILA) method is proposed to mitigate interference on device to device receivers for uplink and downlink resource sharing.

Since full-duplex radios are available for system with small transmit power, and D2D is for short-range communications with small transmit power, studying the full-duplex requirements to be implemented in device to device communication is necessary. The amount of self-interference cancelation in full-duplex radios that is needed for D2D communication under different scenarios should be studied [15]. In view of the above, it becomes essential to focus on the full-duplex aspect of full-duplex D2D communication and study the possibility of implementing already designed fullduplex radios in D2D communication. Interference effects due to sharing radio resources are investigated and also uplink and downlink resource sharing are considered. Knowing the amount of self-interference cancelation required for device to device communication will make it possible to consider fullduplex radios in future standards of wireless systems.

To present a simplified graphical concept of the proposed research work, a conceptual block diagram is developed as presented in figure 2 .

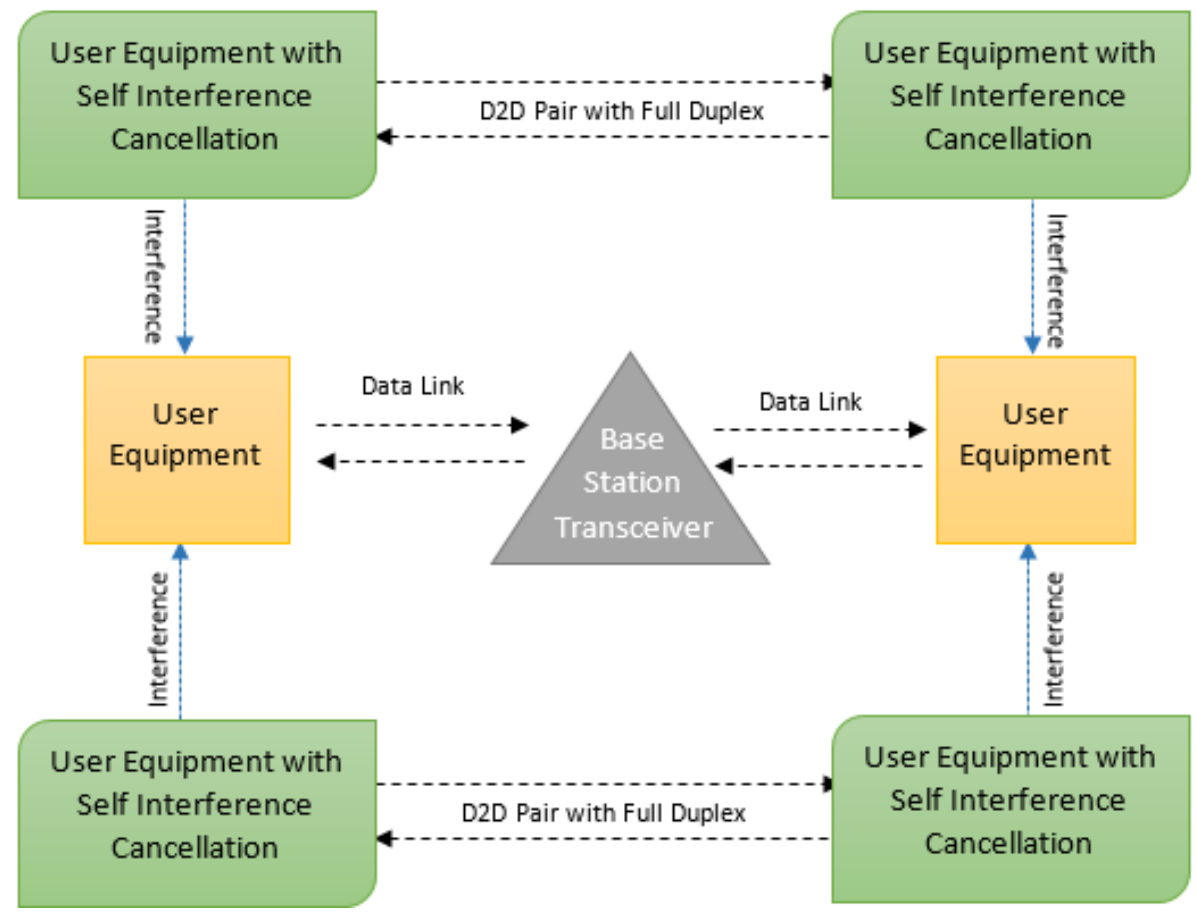

Figure 2. Conceptual Block Diagram of FD-D2D Communication.

Figure 3 shows a heterogeneous networks utilizing a mix of femto, pico, macro and relay cells.

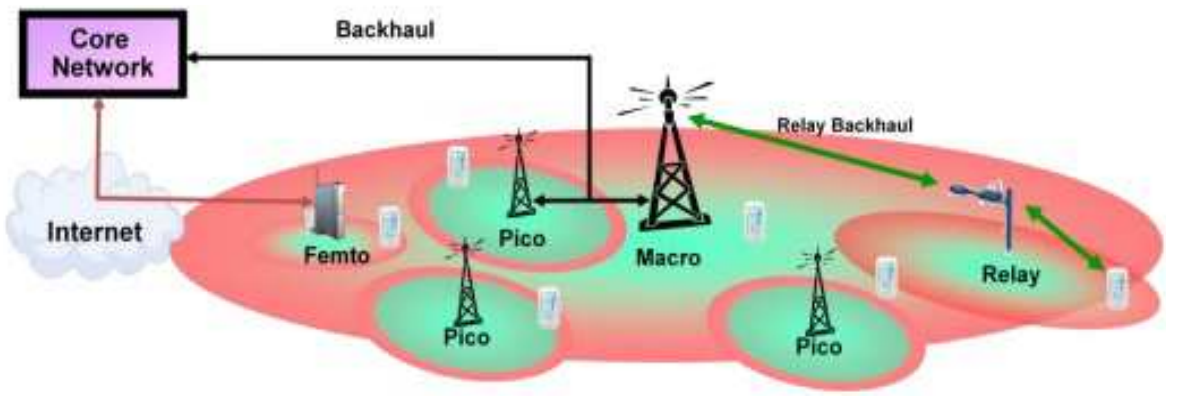

Figure 3. Heterogeneous Networks Utilizing a mix of Femto, Pico, Macro and Relay cells [22].

Figure 4 shows a Heterogeneous Cellular Network with Full duplex D2D communication considered. The diagram shows how the scheme of combining Full duplex radios in a D2D communication underlying a cellular network. 


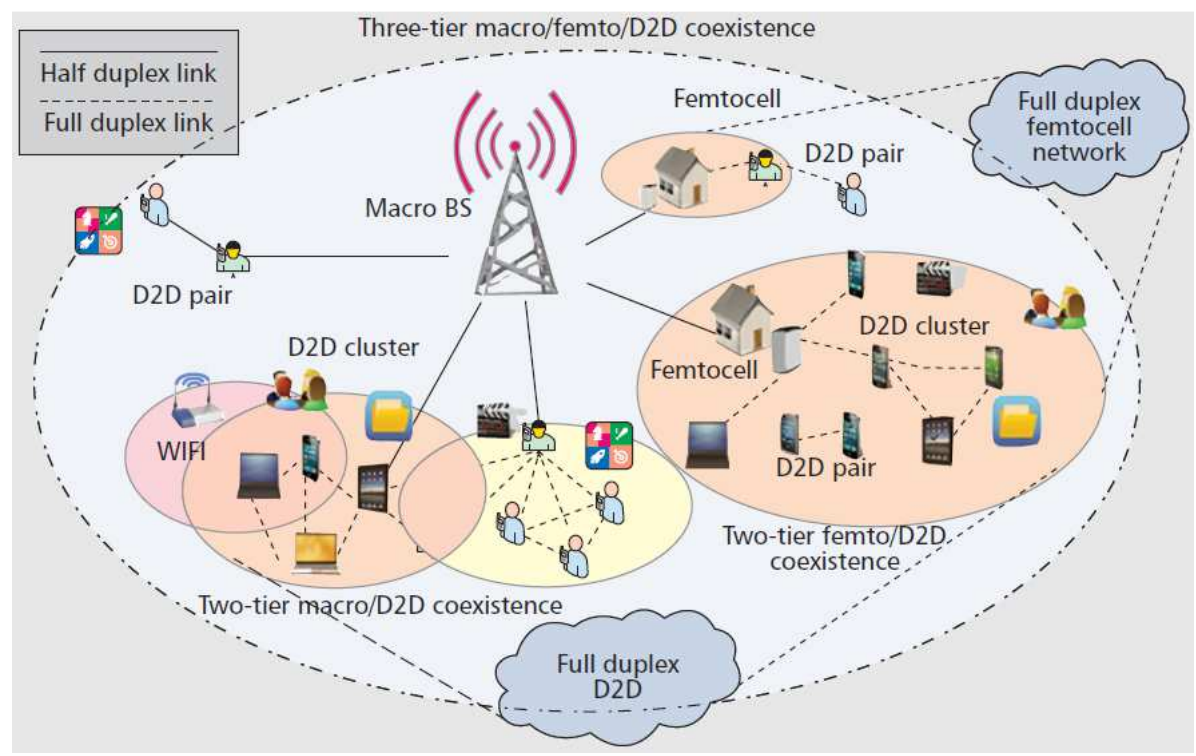

Figure 4. Heterogeneous Cellular network with FD-D2D consideration [16-18].

\section{Aim and Objectives}

The aim of this dissertation is to improve on the spectral efficiency of full-duplex radios in D2D communication in cellular network.

To achieve this aim, the following objectives were set out:

a. To evaluate the main characteristics of D2D communication including its usage, architecture and technical features.

b. To study the applicability of in-band full-duplex radios in $\mathrm{D} 2 \mathrm{D}$ communication.

c. To investigate the performance of system while device to device link is operating in half duplex and in full duplex.

d. To integrate a power control method for D2D pair to limit the interference.

e. To evaluate the performance of full duplex D2D in comparison with half duplex D2D for doubled spectral efficiency.

\section{Modelling and Simulation}

Matlab software was used simulate the model environment for implantation of the developed schemes. The implementation was done in phases, first the general algorithm of Cellular network with Half-duplex and fullduplex device to device communication link was performed, and the results compared. To further analyze the system with full-duplex capabilities, Power control was adopted to manage interference between device to device and base station, interference Limited area method was used to mitigate interference between D2D and Cellular users. The simulation results are presented in graph to ease comparison.

To investigate the applicability of Full-duplex radio in D2D communication underlying a cellular network, since long term evolution systems employ orthogonal frequency division multiple access (OFDMA) in downlink communication, and single carrier frequency division multiple access (SC-FDMA) in uplink, the bandwidth in LTE is divided into resource block (RB). Each RB occupies 180 $\mathrm{KHz}$ in frequency domain equivalent to $0.5 \mathrm{~ms}$ in time domain [19-28]. The minimum uplink scheduling interval in LTE is $1 \mathrm{~ms}$, the smallest resource in frequency domain is two resource blocks, i.e. $360 \mathrm{kHz}$ [29-31]. Here we consider a single cell scenario where 30 users are randomly dropped in the cell, two users with less than 25 meters distance are selected as D2D pair. It is assumed that each user has two LTE resource blocks to communicate in uplink and D2D users have to use sub-channels of other cellular users. Table 1 shows a summary of simulation parameters adopted.

Table 1. General FD-D2D simulation parameters.

\begin{tabular}{ll}
\hline Parameter & Value \\
\hline Cell Radio & $500 \mathrm{~m}$ \\
Maximum D2D Distance & $25 \mathrm{~m}$ \\
CUs per Cell (M) & 30 \\
$\delta_{B}$ & 0.01 \\
$\delta_{I L A}$ & 0.01 \\
$\alpha$ & 4 \\
Maximum CU transmit Power & $23 \mathrm{dBm}$ \\
Noise figure at BS & $2 \mathrm{~dB}$ \\
Noise figure at CU & $9 \mathrm{~dB}$ \\
D2D pathloss Model & $148+40 \log (\mathrm{d}[\mathrm{km}])$ \\
BS to CU Path Loss Model & $128.1+36.7 \log (\mathrm{d}[\mathrm{km}])$ \\
Noise Spectral Density & $-174 \mathrm{dBm} / \mathrm{Hz}$ \\
\hline
\end{tabular}

The overall performance of D2D communication was evaluated using criteria like operating cell; either single cell and multi-cell to obtain a measure of HD-D2D compared to FD-D2D when interference mitigation schemes developed are adopted; evaluation of link performance when the distance between the D2D is increasing, as device to device communication is hinged on proximity, however distance are not fixed. This simulation shows level of Self-Interference cancellation that produces a better result for D2D communication and importantly presents a comparison on the 
spectral efficiency of half-duplex D2D and Full-duplex D2D as a measure of system throughput. The simulation parameters used is in table 2 .

Table 2. Overall Simulation Parameters.

\begin{tabular}{ll}
\hline Parameter & Value \\
\hline Cell Radios & $500 \mathrm{~m}$ \\
Number of cells & 19 \\
Maximum D2D distance & $25 \mathrm{~m}$ \\
CUs per cell & 30 \\
$\delta_{I L A}$ & 0.01 \\
$\alpha$ & 4 \\
Noise figure at BS & $2 \mathrm{~dB}$ \\
Noise figure at CU & $9 \mathrm{~dB}$ \\
D2D pathloss Model & $148+40 \log (\mathrm{d}[\mathrm{km}])$ \\
BS to CU pathloss model & $128.1+36.7 \log (\mathrm{d}[\mathrm{km}])$ \\
Noise spectral density & $-174 \mathrm{dBm} / \mathrm{Hz}$ \\
\hline
\end{tabular}

Simulation Algorithm

Algorithm I: General Simulation Algorithm

Set simulation Environment.

Set simulation Parameters

For $H D-D 2 D$;

$$
R_{T, H D}=R_{C}+R_{C j, H D}+R_{D, H D}
$$

Compute $F D-D 2 D$

$$
R_{T, F D}=R_{C}+R_{C j, F D}+R_{D, F D}
$$

Determine $R_{C} ; R_{C j, H D} ; R_{C j, F D}$

Calculate;

$$
\begin{gathered}
R_{c}=\sum_{i=1, i \neq j}^{M} \log _{2}\left(1+\gamma_{i}\right) \\
R_{c j, H D}=\sum_{j=1}^{K} \log _{2}\left(1+\gamma_{j, H D}\right) \\
R_{c j, F D}=\sum_{j=1}^{K} \log _{2}\left(1+\gamma_{j, F D}\right) \\
\text { For } M=1, K=8
\end{gathered}
$$

Estimate $S I N R$ for $D 1 ; \gamma_{D 1}=R_{D, H D}$ and $R_{D, F D}$

$$
\begin{aligned}
& R_{D, H D}=\log _{2}\left(1+\gamma_{D 1}\right) \\
& R_{D, F D}=\sum_{l=1}^{2} \log _{2}\left(1+\gamma_{D l}\right)
\end{aligned}
$$

Interference from $D 2 D$ on $B S$

$$
\gamma_{i}=\frac{P_{c i} \cdot G_{c i, B S}}{N_{0}}
$$

Determine Overall SINR

$$
\begin{gathered}
\text { for } H F-D 2 D \text { and } F D-D 2 D \\
\gamma_{j, H D}=\frac{P_{c i} \cdot G_{c i, B S}}{N_{0}+I_{D 2, c j}} \\
\gamma_{j, F D}=\frac{P_{c i} \cdot G_{c i, B S}}{N_{0}+I_{D 1, c j}+I_{D 2, c j}}
\end{gathered}
$$

Generate $N_{o}$ (White Gaussian Noise additive)

\section{Estimate Residual SI at Node $=I_{l}$}

Calculate $\gamma_{1}$

$$
\begin{gathered}
H D-D 2 D \gamma_{1}=\frac{P_{D 2} \cdot G_{D}}{N_{0}+\sum_{j=1}^{K} I_{D 2, c j}} \\
F D-D 2 D \gamma_{1}=\frac{P_{D 2} \cdot G_{D}}{N_{0}+I_{l}+\sum_{j=1}^{K} I_{c j, l}} \\
\text { Save } R_{c} ; R_{D, H D} ; R_{c j, F D}
\end{gathered}
$$

Algorithm II: Power Control Scheme

Set simulation parameters.

Compute ISR at base:

$$
I S R=\frac{P_{D l} \cdot G_{l, B S}}{P_{R C}} \leq \delta_{B}
$$

Determine $P_{D l}$

Define SINR of $B S=\delta_{B}$

$$
\text { select user lth }
$$

If $P_{D l} \leq \frac{P_{R C} \cdot \delta_{B}}{G_{l, B S}}$

Calculate channel gain $=G_{l, B S}$

Set $P_{R C}$

Save all users with $P_{D}<\delta_{B}$

The power control scheme is implemented over two separate scenarios, D2D sharing resource with only one cellular user and when resource of multiple cellular users is being shared. To observe the effect of residual SI on SINR of the D2D, comparison between the performance of HD-D2D and FD-D2D is carried out for Single Cell and Multi-cell scenarios. For each scenario, the SI cancellation is estimated at between $65 \mathrm{~dB}$ and $110 \mathrm{db}$.

Algorithm III: Interference Limited Area

Set simulation parameters.

\section{Determine radius $d_{i}$}

Select D2D user $D_{i}$ compute $\delta_{I L A}$

If $d_{l}<\left(\frac{P_{c m a x} \cdot G_{0}}{P_{D l} \cdot G_{D} \cdot \delta_{I L A}}\right)^{1 / \alpha}$ select $D_{i}$; else,

$$
\text { select } i+1
$$

If $d_{l}<\left(\frac{P_{c \max } \cdot G_{0}}{P_{D l+1} \cdot G_{D} \cdot \delta_{I L A}}\right)^{1 / \alpha}$ select $D_{i+1}$

$$
\text { List selected } D_{i}
$$

Set $d_{l}$ minimum distance Compute channel gain $G_{0} \cdot\left(d_{l}\right)^{-\alpha}$ for $G_{c i, D l} i=1+1$;

$$
\text { Determine } G_{c i, D l}>\frac{P_{D l} \cdot G_{D} \cdot \delta_{I L A}}{P_{c \max }}
$$

$$
P_{\text {cmax }} \text { max.transmitt Power }
$$

Save $d_{l}, G_{c i, D l}$.

The Interference Limited Area method considering the 
minimum Signal to interference plus noise ratio (SINR) requirements for cellular users, calculation of the interference-over-signal ratio for cellular user is performed, also considering the maximum D2D transmit power. This ISR is set to be smaller than the predefined threshold. The group of users selected must meet the lower ISR criteria so that their sub-channel can be used in D2D link without harmful interference effect on the network cell. The maximum transmits power of the cellular user and pathloss exponent $P_{\max }$ and $\alpha$ respectively are define in the simulation parameters.

\section{Results and Discussions}

Figure 5 shows the result obtained for the general system simulation to show effectiveness of using FD-D2D in a cellular network. The result in figure 5 shows the throughput of the system when only Cellular user $\mathrm{j}\left(\mathrm{CU}_{\mathrm{j}}\right)$ is sharing resource with D2D link and figure 6 when multiple users sharing resource with D2D. From the comparison of cellular only, HD-D2D and FD-D2D links, at less than $75 \mathrm{~dB}$ selfinterference cancellation, the HD-D2D out performs the FDD2D but compared to cellular FD-D2D has tremendous impact on throughput improvement on the cellular network in both cases, the minimum Signal to noise ratio (SNR) at BS is kept at $10 \mathrm{~dB}$. As the SI cancellation increases, the performance of FD-D2D also increase and it out performance HD-D2D. The significance of the result is that as Selfinterference (SI) cancellation only one user shares resource with D2D, the amount of Residual Self-Interference in high, therefore degrades throughput, but when multiple users share resources, the SI cancellation improves, residual SI will decrease causing Signal to interference plus noise ratio (SINR) of D2D receivers to increase making FD-D2D outperform cellular only and HD-D2D.

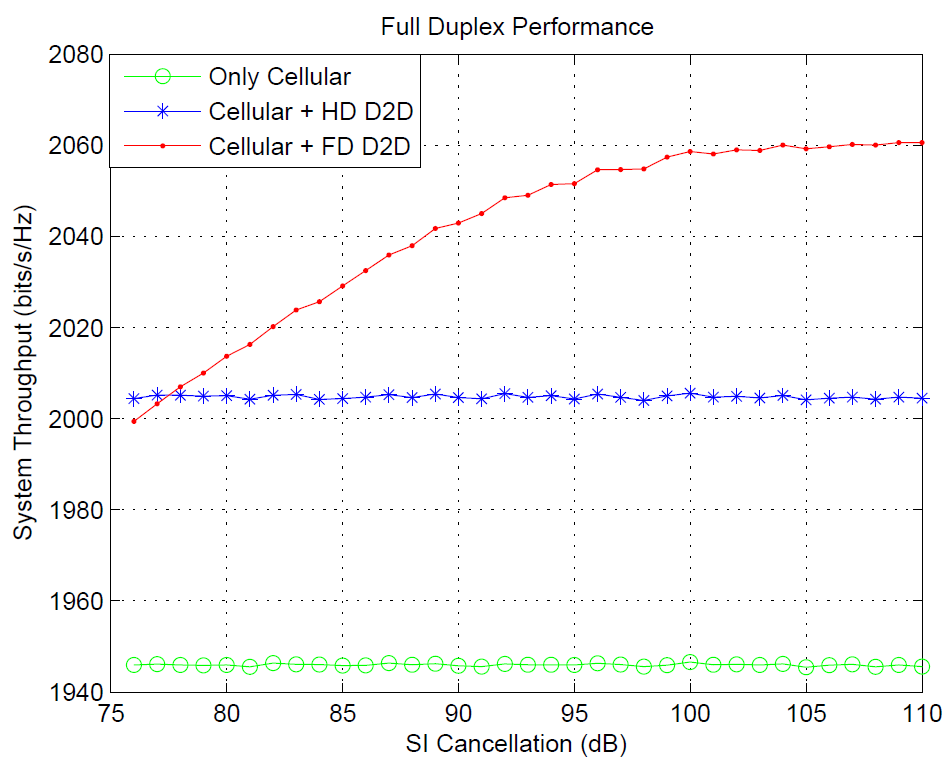

Figure 5. General system throughput with one user sharing resource with D2D link.

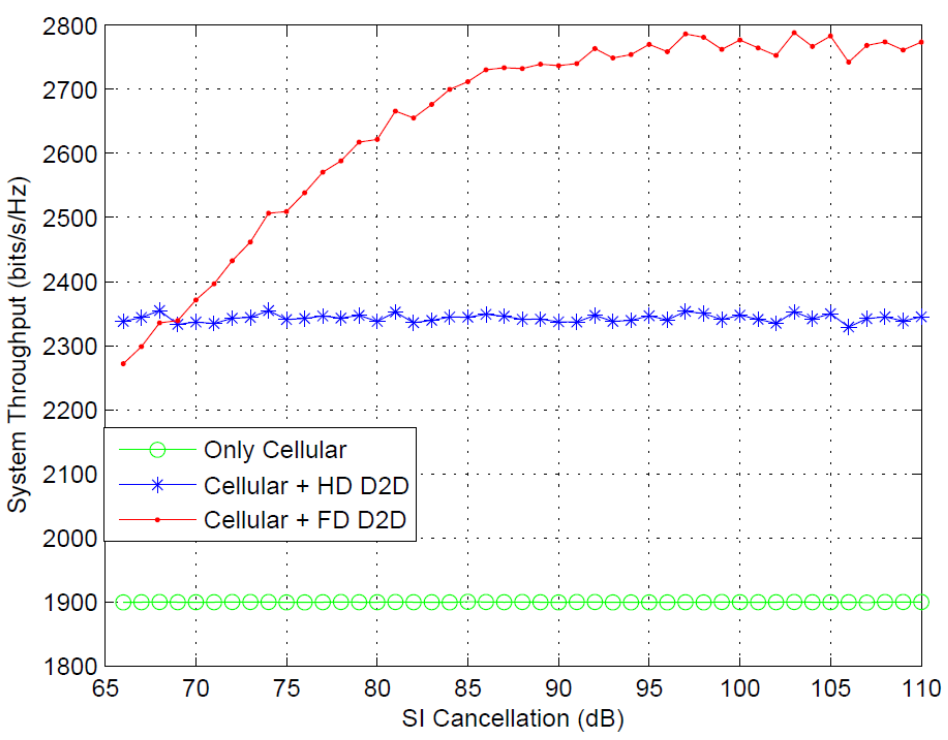

Figure 6. General system throughput with multiple user sharing resource with D2D link. 


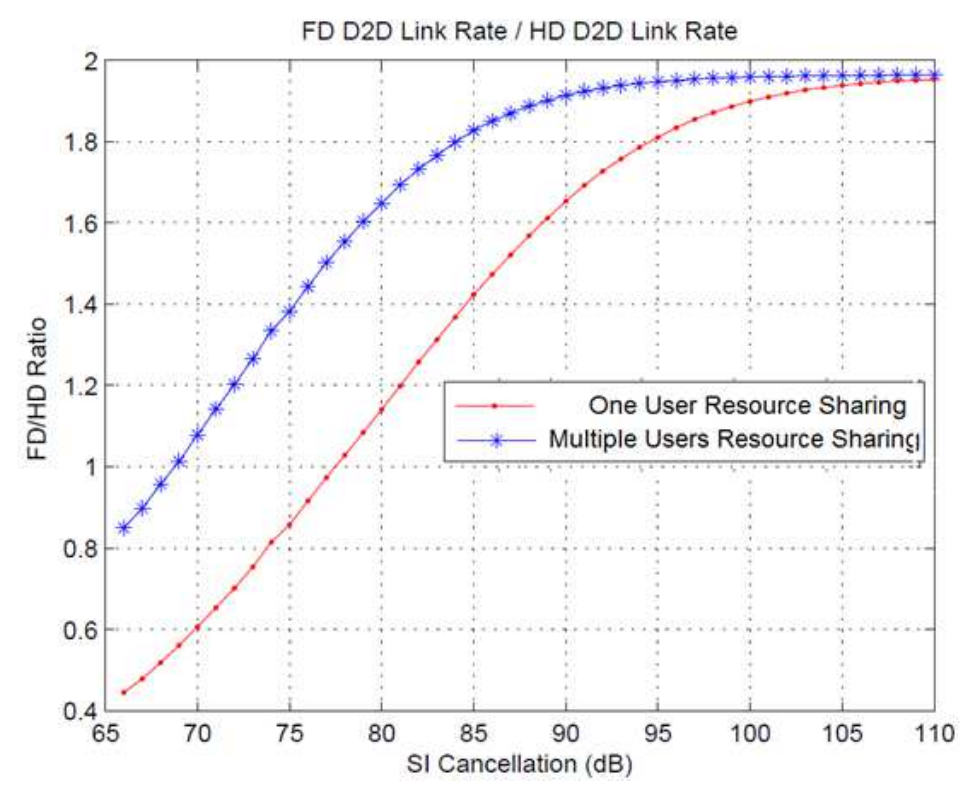

Figure 7. Full-Duplex/Half-Duplex link rate comparison with power control scheme.

The result presented in figure 7 is shown in ratio of FDD2D link rate over HD-D2D link rate for two scenarios. The first is when only one cellular user is sharing uplink resource with D2D receiver and when multiple cellular users are sharing resource with D2D link, the power control scheme is implemented for both scenarios. The Half-duplex D2D shows a better performance at SI cancellation of between 65 to 78 $\mathrm{dB}$ when only one user is sharing resources, however, the Full-duplex D2D shows significant link rate performance when multiple users share resource SI cancellation of as low as $69 \mathrm{~dB}$. This result indicates that FD-D2D suffer performance degradation at low SI cancellation levels and especially so when resource is being shared with only on cellular user.

Interference limited area method was implemented, and the result obtained is shown in figure 8. The figure shows full-duplex D2D over half-Duplex D2D ratio versus the SNR target at BS for uplink and downlink signals at different selfinterference cancellation amount.

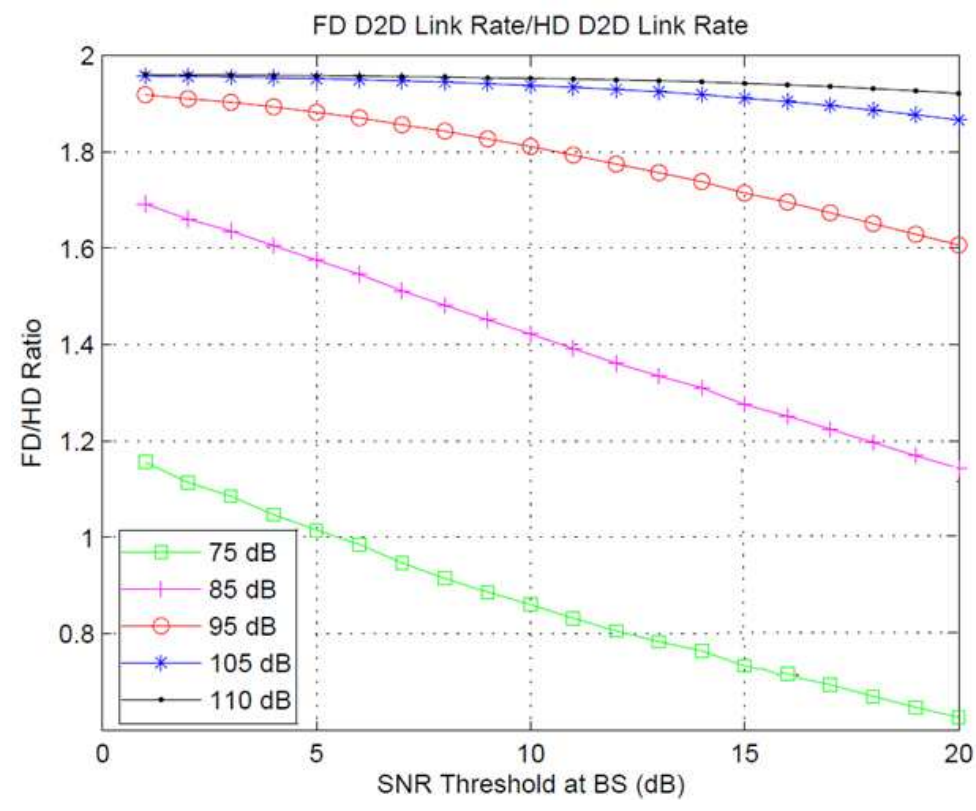

Figure 8. Full-Duplex/Half Duplex link rate comparison for different SINR Target.

The results are significant as is indicates that as SINR target increases, cellular users need to transmit with higher power level, this results in larger transmit power for D2D users also. The increase in transmit power demand causes increase in residual self-interference [32]. Therefore, as SNR target increases, Fullduplex D2D communication performance decreases, due to a degrading self-interference cancellation. The same applies to HD-D2D it is more affected compared to FD-D2D. 


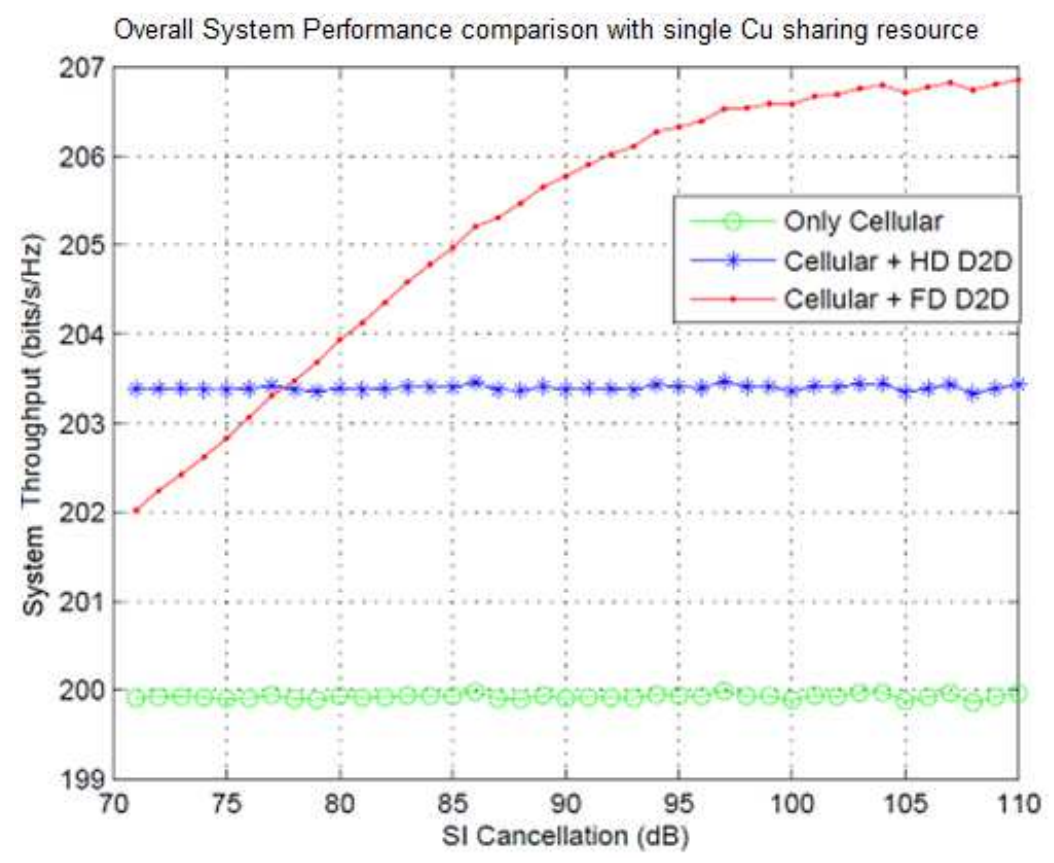

Figure 9. Overall system performance comparison for single CU resource sharing.

The overall performance of the D2D system is simulated, the results obtained gives a measure of how each of the two-radio system enhance the performance of D2D network. To evaluate their performance, the simulation parameters are set the same for both HD and FD D2D [33-35], the interference management schemes developed is deployed for both radio implementations. From figure 9, it is noted that the performance of cellular only communication is unaffected with the self-interference cancellation mechanism between 70 to $110 \mathrm{~dB}$, throughput remains fairly constant for increasing value of SI cancellation. The performance of HD-D2D is observed to be better than FDD2D at low values of SI cancellation below $78 \mathrm{~dB}$, this is so because at low values of SI cancelation, the level of residual self-interference on full-duplex radios is too high and causes deterioration of the SINR of D2D receivers.

As the number of cellular resource increases, the performance of FD-D2D increase significantly as the throughput outperforms HD-D2D at SI cancellation of $73 \mathrm{~dB}$ compared to about $78 \mathrm{~dB}$ in single $\mathrm{CU}$ operation as depicted in figure 10. At $110 \mathrm{~dB}$ self-interference cancellation on the FD-D2D, the throughput performance $(\sim 3030$ bits $/ \mathrm{Hz})$ doubles that of HD-D2D ( $\sim 3090$ bits/Hz). This result shows that theoretical expectation of achieving double spectral efficiency is achieved.

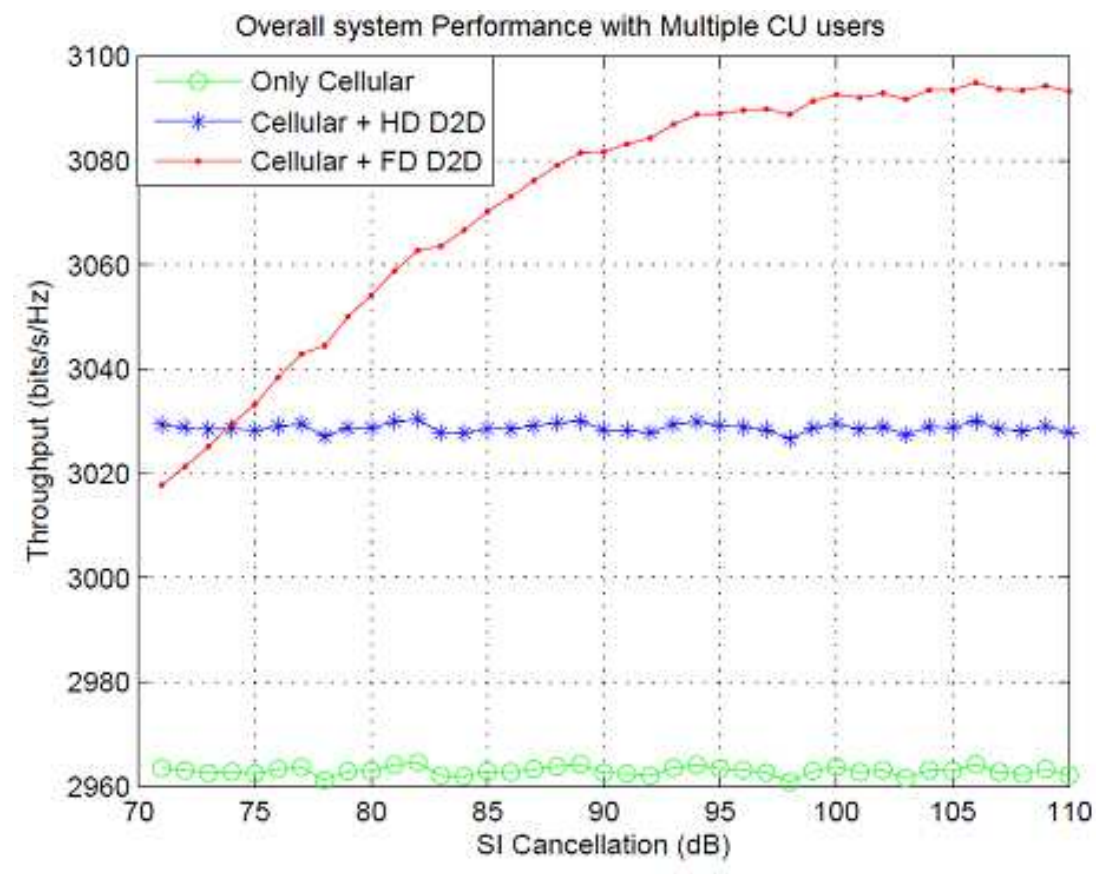

Figure 10. Overall system performance comparison for Multi CU resource sharing. 


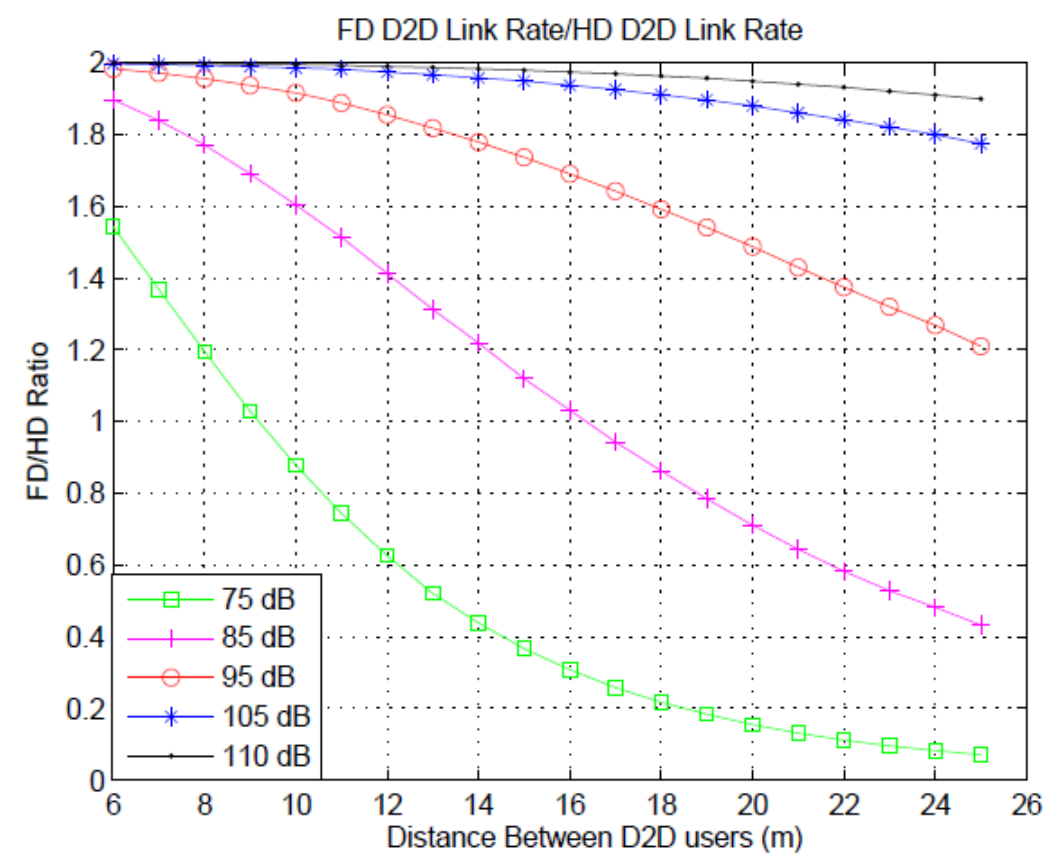

Figure 11. FD/HD Throughput ratio based on D2D distance.

One very important aspect of D2D communication is proximity, small distance between D2D users. To evaluate the performance of the implemented scheme, the effect of the distance D2D users from each other on the overall performance of Full duplex radio is an important evaluation. The result in figure 11 presents ratio of FD-D2D throughput to that of HD-D2D throughput for 5 levels of selfinterference cancellations, based on the distance between D2D users.

From figure 11, it can be deduced that distance between D2D has a significant effect on the system throughput performance. As the distance between the D2D increase, FDD2D throughput deteriorates this is due to increase in residual Self-interference at a distance of 24 meters, and SIcancellation level at less than $85 \mathrm{~dB}$ the throughput of HDD2D outperformance FD-D2D. However significant throughput performance increase is observed when the SIcancellation is above $85 \mathrm{~dB}$. Very significant to note from the result in figure 11, at SI-cancellation above $110 \mathrm{~dB}$, FullDuplex Device to Deice communication shows almost double the throughput of half-duplex D2D.

\section{Conclusion}

This research simulated the use of full-duplex radios in D2D communication, underlying a cellular network. The practicality and Self-interference cancellation requirements necessary for implementation of FD radios in D2D communication was investigated, for currently available FD radios with SI cancellation of up to $100 \mathrm{~dB}$. Research and simulations results shows that current available FD radios are practically implementable in D2D communication links. For the self-Interference cancellation requirement challenges, two interference mitigation schemes were implemented and were combined to manage self-interference in FD-radio for D2D communication. The use of power control scheme to mitigate interference between D2D and Base station was combined with Interference limited area method to manage interference between cellular devices and D2D pairs in the network. Implantation was carried out for different scenarios and under varying levels of SI-cancellation and in comparison, between Half-duplex D2D and full-Duplex D2D to evaluate their performance. The results obtain clearly shows significant performance for FD-D2D communication, with FD-D2D out performing HD-D2D in all areas where the SIcancellation is above $100 \mathrm{~dB}$. The throughput performance of FD-D2D indicates a doubling of the spectral efficiency of the overall system. A study of the effect of D2D link distance shows that at $110 \mathrm{~dB}$ SI-cancellation, FD-D2D provides almost twice the throughput of HD-D2D. The overall result indicates that FD-D2D has a promising future in the quest to provide increasing service and better spectrum utilization.

\section{References}

[1] A. Goldsmith, Wireless Communications. New York, NY, USA: Cambridge University Press, 2005.

[2] R. Zhang, X. Cheng, L. Yang, and B. Jiao, "Interference-aware graph-based resource sharing for device-to-device communications undying cellular net- works," in Wireless Communications and Networking Conference (WCNC), 2013 IEEE, April 2013, pp. 140-145.

[3] D. Bharadia, E. McMilin, and S. Katti, "Full duplex radios," SIGCOMM Comput. Commun. Rev., vol. 43, no. 4, pp. 375386, Aug. 2013.

[4] D. Bharadia and S. Katti, "Full Duplex MIMO Radios," in 11th USENIX Symposiumon Networked Systems Design and Implementation (NSDI 14), Seattle, WA, Apr. 2. 2014. 
[5] L. Liu, and T. Yuanwei. (2016). "Secure D2D communication in large-scale cognitive cellular networks: a wireless power transfer model”. IEEE Trans. Commun., pp. 65-76, Aug. 2016.

[6] K. Doppler, M. Rinne, C. Wijting, C. Ribeiro, and K. Hugl, "Device-to-device communication as an underlay to LTEadvanced networks," Communications Magazine, IEEE, vol. 47, no. 12, pp. 42-49, Dee 2009.

[7] K. Doppler, M. Rinne, P. Janis, C. Ribeiro, and K. Hugl, "Device-to-Device Communications; Functional Prospects for L1c-Advanced Networks," in Communications Workshops, 2009. ICC Workshops 2009. IEEE International Conference on, June 2009, pp. 1-6.

[8] D. Wang and X. Wang, "An interference management scheme for device-to- device multicast in spectrum sharing hybrid network," in Personal Indoor and Mobile Radio Communications (PIMRC), 2013 IEEE 24th International Symposium on, Sept 2013, pp. 3213-3217.

[9] T. Peng, Q. Lu, H. Wang, S. Xu, and W. Wang, "Interference avoidance mechanisms in the hybrid cellular and device-todevice systems," in Personal, Indoor and Mobile Radio Communications, 2009 IEEE 20th International Symposium on, Sept 2009, pp. 617-621.

[10] J. Gu, S. J. Bae, B.-G. Choi, and M. Y. Chung, "Dynamic power control mechanism for interference coordination of device-to-device communication in cellular networks," in Ubiquitous and Future Networks (ICUFN), 2011 Third International Conference on, June 2011, pp. 71-75.

[11] S. Breuck, "Heterogeneous networks in LTE-Advanced". 8th International Symposium on Wireless Communication Systems, ISWCS 2011, Aachen, Germany.

[12] L. Wang, F. Tian, T. Svensson, D. Feng, M. Song and S. Li; "Exploiting full duplex for D2D communication in heterogeneous Networks" IEEE Communication magazine, May 2015. Pp- 146-152.

[13] P. Gandotra, and R. K. Jha, "Device-to-Device Communication in Cellular Networks: A Survey" Journal of Network and Computer Applications, Feb. 2016, pp. 99-117.

[14] S. Goyal, P. Liu, S. Hua, S. Panwar, "Analyzing a full-duplex cellularsystem," Information Science and Systems (CISS 2013), Baltimore, Mar. 2013.014, pp. 359-372.

[15] Yang, C., Li, J., \& IGI Global. (2016). Game theory framework applied to wireless communication networks (pp. PDFs (502 pages)). Retrieved from http://services.igiglobal.com/resolvedoi/resolve.aspx?doi=10.4018/978-1-46668642-7. On 15/2/18.

[16] Ghazanfari, A. (2014). Coordinated Beamforming and Power Control for Network Controlled Device-to-Device (D2D) Communication. (Msc Master's Thesis), University of Oulu.

[17] Zhang, Z., Wang, L., \& Zhang, J. (2017). Energy Efficiency of D2D Multi-User Cooperation. Sensors (Basel), 17 (4). Pp doi: $10.3390 / \mathrm{s} 17040697$.

[18] B. Mate. (2009). Unicast Communication in Vehicular ad-hoc Networks: A Reality Check. Communications Letters, IEEE, 13 (12): 995-997, Dec. 2009. ISSN 1089-7798. doi: 10.1109/LCOMM.2009.12.091497.

[19] Kaur, G P., Birla, J., Ahlawat, J. (2011). Generations of Wireless Technology. International Journal of Computer
Science and Management Studies, Vol. 11, Issue 02 pp 435-441.

[20] G. Patil, and P. Wankhade, "5G Wireless Technology" International Journal of Computer Science and Mobile Computing, Vol. 3, Issue. 10, October 2014, pg. 203-207.

[21] S. Amir, and M. Shikh-Bahaei. "Multi-user time frequency downlink scheduling and resource allocation for LTE cellular systems." Wireless Communications and Networking Conference (WCNC), 2010 IEEE. IEEE, 2010.

[22] K. Jha, G. Patel, "Device-to-Device Communication in Cellular Networks: A Survey" Journal of Network and Computer Applications, Feb 2016, 71, 99-117.

[23] J. Kim, N. A. Karim, A., \& S. Cho, "An Interference Mitigation Scheme of Device-to-Device Communications for Sensor Networks Underlying LTE-A. Sensors" Wireless Communications and Networking Conference (Basel) June 2017, 17 (5).

[24] R. Kreher, \& K. Gaenger, "LTE signaling, troubleshooting, and optimization" (pp. 1 online resource). Retrieved from http://search.ebscohost.com/login.aspx?direct=true\&scope=sit $\mathrm{e} \& \mathrm{db}=$ on $01 / 02 / 18$.

[25] Xu, C., Song, L., Han, Z., \& Springer Link (Online service). (2014). Resource management for device-to-device underlay communication Springer Briefs in computer science, (pp. 67- 79.

[26] Sambo, Y A., Shakir, M Z., Héliot, F., Imran, M A., Mumtaz, S., Qaraqe, K A. (2014). Device-to-Device Communication in Heterogeneous Networks. Smart Device to Smart Device Communication pp 219-235.

[27] Masood U., Ghazanfar A. S., Mujahid M., Muhammad A. I., \& Rahim T. (2016). Interference Mitigation in D2D Communication Underlaying LTE-A Network. IEEE 21693536.

[28] S. P. Herath and T. Le-Ngoc, "Sum-rate performance and impact of self-interference cancellation on full-duplex wireless systems," in Personal Indoor and Mobile Radio Communications (PIMRC), 2013 IEEE 24th International Symposium on, Sept 2013, pp. 881-885.

[29] E. Everett, M. Duarte, C. Dick, and A. Sabharwal, "Empowering full-duplexwireless communication by exploiting directional diversity," in Signals, Systems and Computers (ASILOMAR), 2011 Conference Record of the Forty Fifth Asilomar Conference on, Nov 2011, pp. 2002-2006.

[30] J. I. Choi, M. Jain, K. Srinivasan, P. Levis, and S. Katti, "Achieving single channel, full duplex wireless communication," in Proceedings of the Sixteenth Annual International Conference on Mobile Computing and Networking, ser. MobiCom'10. New York, NY, USA: ACM, 2010, pp. 1-12.

[31] M. Jain, J. I. Choi, T. Kim, D. Bharadia, S. Seth, K. Srinivasan, P. Levis, S. Katti, and P. Sinha, "Practical, realtime, full duplex wireless," in Proceedings of the 17th Annual International Conference on Mobile Computing and Networking, ser. Mobi Com '11. New York, NY, USA: ACM, 2011, pp. 301-312.

[32] E. Aryafar, M. A. Khojastepour, K. Sundaresan, S. Rangarajan, and M. Chiang, "MIDU: Enabling MIMO Full Duplex," in Proceedings of the 18th Annual International Conference on Mobile Computing and Networking, ser. Mobicom '12. New York, NY, USA: ACM, 2012, pp. 257-268. 
[33] M. Duarte and A. Sabharwal, "Full-duplex wireless communications using off-the-shelf radios: Feasibility and first results," in Signals, Systems and Computers (ASILOMAR), 2010 Conference Record of the Forty Fourth Asilomar Conference on, Nov 2010, pp. 1558-1562.
[34] M. Duarte, C. Dilk, and A. Sabharwal, "Experiment-driven characterization offull-duplex wireless systems," IEEE Transactions on Wireless Communications, vol. 11, no. 12, pp. 4296-4307, December 2012. 